\title{
The principle of the isosceles triangle for geometric inequalities
}

\begin{abstract}
Razvan Alin Satnoianu
Razvan A. Satnoianu obtained his MSc in applied mathematics at the University of Bucharest in 1992 and his PhD in mathematics at the University of Leeds in 1998. He then worked as a postdoctoral fellow in mathematical biology at Oxford University. Presently, he is a permanent faculty member in the School of Engineering and Mathematical Sciences at the City University in London. His main fields of interest are geometric and analytic inequalities, pattern formations, reaction-diffusion equations and their applications in biology and chemistry.
\end{abstract}

\section{Introduction}

In a recent note published in [8] we have presented a universal yet simple and unexpected method of proof for geometric inequalities in an euclidian triangle. The method consisted of reducing the case for general triangles to that of isosceles triangles. The idea went as follows. For a given euclidean triangle denote by $a, b, c$ the measures of its angles in radians chosen so that

$$
0 \leq c \leq b \leq a \leq \pi \quad \text { and } \quad a+b+c=\pi .
$$

Then, to establish that some inequality $f(a, b, c) \geq 0$ holds, it suffices to show that

$$
f(a, b, c) \geq f\left(a, \frac{b+c}{2}, \frac{b+c}{2}\right) \geq 0,
$$

Ein Problemkreis der Elementargeometrie besteht in der Charakterisierung typischer Grössen eines Dreiecks, wie z.B. des In- oder Umkreisradius, durch Ungleichungen. In der vorliegenden Arbeit wird die Lösung dieser analytisch-geometrischen Problemstellung dahingehend systematisiert, dass eine hinreichende Bedingung gegeben wird, welche es erlaubt, den Beweis solcher Ungleichungen für beliebige Dreiecke auf die entsprechende Beweisführung für die Klasse gleichschenkliger Dreiecke zurückzuführen. Dieses Vorgehen wird im zweiten Teil der Arbeit anhand einiger konkreter Beispiele illustriert. 
where the second inequality represents the case of an isosceles triangle. At first sight this looks like a complication as it is not a direct way to solve the problem. Yet in [8] we have shown, by exploring some suggestive examples, that it often proves easier to establish the two inequalities at (1.2) than to establish the original one directly. Anybody reading the result in [8] would naturally be lead to consider what is the underlying structure of the triangle geometry that allows for such a simple property in the class of geometric inequalities for triangles.

It is the purpose of this paper to establish a general result, which gives a simple sufficient condition that answers the question above. In essence this establishes when "the set of values taken by a function defined on the class of all euclidean triangles is exactly equal with the set of values taken by the same function when restricted to only the subset of isosceles triangles". The importance of the present result lies in reducing the complicated problem of analysis of the variation of a three variable function (representing the given inequality) to that of a single variable function, which is an elementary task. We call this main result "The principle of the isosceles triangle" or PIT in short. This is presented in the first part. The method has a wide range of applications, which are illustrated in the second part (for example the vast majority of the book [4] can be treated this way). We conclude by alluding to the universality of the principle of the isosceles triangle as possibly being a universal method of analysis in triangle geometry.

\section{The main results}

In order to state the main result we shall specify the class of functions to which it applies. Naturally, we would like to be able to address at least any geometric inequality related to the fundamental triangle inequality (see $[4$, p. 2] for details). The formal result is very simple but forms the basis of the discussion in the sequel.

Lemma (The principle of the isosceles triangle - PIT) Assume that ABC is a triangle for which (1.1) holds and let $g=g(a, b, c)$ be a differentiable function in $T=\{(a, b, c)$ | $a, b, c \geq 0, a+b+c=\pi\} \subset \mathbb{R}^{3}$ with real values. Let also $T^{i}$ be the subset of $T$ formed by all isosceles triangles. Suppose that

$$
g \geq 0 \text { for all triangles in } T^{i}
$$

and there is at least one isosceles triangle $T_{0}^{i}$ on which $g$ attains a global minimum in $T$. Then $g(a, b, c) \geq 0$ in $T$.

Proof. Clearly $T$ is a compact domain in $\mathbb{R}^{3}$. It follows that $g$ is bounded and attains its bounds on $T$. From the hypotheses of the theorem we have that $g\left(T^{\ell}\right) \geq g\left(T_{0}^{i}\right) \geq 0$ for every triangle $T^{\ell} \in T$ and that ends the proof.

Definition A function $g$ with the property that there is at least one isosceles triangle $T_{0}^{i}$ which realises a global minimum for $g$ in $T$ will be said to have the property of minimum isosceles extrema. Analogously, we define the property of maximum isosceles extrema. A function with the property of minimum isosceles extrema and maximum isosceles extrema will be said to have the property of the global isosceles extrema. 


\section{Remarks}

1. The following example shows that both hypotheses in the above lemma are essential. Consider the function

$$
f(a, b, c)=(a-x)^{2}+(b-y)^{2}+(c-z)^{2}
$$

where $a, b, c \geq 0, a+b+c=\pi$ and $x, y, z \geq 0, x+y+z=\pi$. Clearly, $(x, y, z)$ is a global minimum. Take for example $x=1, y=2, z=\pi-3$. Then, $f(1,2, \pi-3)=0$. Also one readily finds that $f(t, t, \pi-2 t)$ has its minimum at $\frac{(x-y)^{2}}{2}=\frac{1}{2}>0$.

A simple example of a symmetric function in $(a, b, c)$ can also be constructed. Take the function

$$
\begin{aligned}
f_{1}(a, b, c)=(a-x)^{2}(a-y)^{2}(a-z)^{2} & +(b-x)^{2}(b-y)^{2}(b-z)^{2} \\
& +(c-x)^{2}(c-y)^{2}(c-z)^{2}
\end{aligned}
$$

for $a, b, c \geq 0, a+b+c=\pi$ and $x, y, z \geq 0, x+y+z=\pi$ with $x>y>z$. Clearly, $f_{1}$ is symmetric in $(a, b, c)$ and has global minimum at points not included in the set of the isosceles triangles.

2. The vast majority of the inequalities in [4], which is the standard reference in the subject of triangle inequalities, do fit however, in the framework given by the above lemma. This will be demonstrated below. One usually checks that for a given functional inequality there is some "easy" case of equality for some particular triangle (e.g., if $T_{0}^{i}$ in the above is equilateral or degenerate isosceles with two right angles or two angles zero, etc.). The reason behind this property lies with the dependence of a typical such inequality on the triangle's elements. Most of the geometric inequalities in [4] contain expressions depending on elements such as altitudes, bisectors, medians, radii, semiperimeter, etc. All these quantities depend in a regular and symmetric way on the triangle's angles. For any such case it is clear that, by using standard results, we can always express these in terms involving only the trigonometric functions of the triangle's angles, which reduces the problem to the case shown above.

The above remarks point to the question of finding a practical characterisation for the class of functions to which the principle of the isosceles triangle inequality can be applied. Property (2.1) is easy to check in practice, as it requires only one variable calculus. The most difficult assumption to verify in applications is the global isosceles extrema property. This of course, requires three variable calculus. We now shall show that this problem can also be reduced to one variable calculus.

Theorem 1 (The reduction property for the principle of the isosceles triangle). Let $g=g(a, b, c)$ be a differentiable function in $T_{\text {open }}=\{(a, b, c) \mid a, b, c>0, a+b+c=$ $\pi\} \subset \mathbb{R}^{3}$ with real values. Then, $g$ has the property of global isosceles extrema if for any pair, e.g. $(a, b)$, the functions $h_{ \pm}(x)=g(a \pm x, b \mp x, c)$ satisfy

$$
\left.\frac{\mathrm{d} h_{ \pm}}{\mathrm{d} x}\right|_{x=0}=0 \quad \text { if and only if } a=b \text {. }
$$

Here, we take $0 \leq x \leq \min \left\{|b-c|, \frac{|a-b|}{2}\right\}$. 
Proof. Suppose that $g$ has the property (2.4). Any point of extremum for $g$ will then be located in the set of values that satisfy $h_{ \pm}^{\prime}(0)=0$. From (2.4) this gives $a=b$ so that $g$ has the global isosceles extrema property.

Remark The case $c=0$ already reduces the problem to one variable calculus.

In the following, we shall call a function of $(a, b, c)$ symmetric if for any pair, e.g. $(a, b)$ we have that $g(a, b, c)=g(b, a, c)$, for all $a, b, c$.

Corollary 1. A symmetric function of $(a, b, c)$ has the property of global isosceles extrema if and only if it has the property of minimum isosceles extrema. A similar result is true if one replaces "minimum" by "maximum" in the above.

The main usefulness of the lemma and Theorem 1 is clearly in their application in finding/validating the range of values of a given function depending on the elements of the triangle. It is also clear that the result will hold even if the function may depend explicitly on more elements than just the triangle's angles.

Corollary 2. Let $g: T \rightarrow \mathbb{R}$ be a differentiable function with the properties of global isosceles extrema (i.e. for both global minimum and global maximum) as in the theorem above. Then, the set of values of $g$ in $T$ is equal to the set of values of $g$ taken when restricted to only the subset of all isosceles triangles in $T$.

Proof. Let $T_{1} \subset T$ be the set of all isosceles triangles. $T_{1}$ is a bounded set and $g$ is a continuous function so there are two real numbers $m \leq M$ such that $g\left(T_{1}\right)=[m, M]$, where $g(T)$ represents the range of $g$ on $T$. Take $g_{1}=g-m, g_{2}=M-g$. Then, $g_{1}, g_{2}$ satisfy the conditions of our theorem and so we deduce that

$$
[m, M]=g\left(T_{1}\right) \subset g(T) \subset[m, M]
$$

which ends the proof.

Corollary 3. The result of Corollary 2 holds true for "vector-valued" functions, i.e. functions of the form $g: T \rightarrow \mathbb{R}^{p}, p \geq 1$ being an integer, which satisfy for each component the properties given in Corollary 2.

Proof. All we have to do is to apply Corollary 1 for each component of $g$ in turn.

Before ending this part we shall present two other sufficient conditions that guarantee that a function $f$ satisfies the global isosceles extrema property. The first one is relation (1.2), which was presented in [8]. It is clear that any such function $f$ does satisfy the minimum isosceles extrema property. When applied to the examples in [8] the new method presented here (Theorem 1) is far more efficient than the method given by relation (1.2) as we shall illustrate in the next section.

A second sufficient condition is the following:

Proposition 1. Suppose that $f$ is a convex function such that

$$
2 f(a, b, c) \geq f(a, a, c)+f(b, b, c)
$$

for all $0 \leq c \leq b \leq a \leq \pi$ and $a+b+c=\pi$. Then, $f$ has the property of minimum isosceles extrema. 
Proof. We shall show that $f$ verifies relation (1.2). The function $f$ being convex it satisfies Jensen's inequality. Therefore, we have that

$$
\begin{aligned}
f\left(\frac{a+b}{2}, \frac{a+b}{2}, c\right) & \leq \frac{1}{2}\left(f\left(a, \frac{a+b}{2}, c\right)+f\left(b, \frac{a+b}{2}, c\right)\right) \\
& \leq \frac{1}{2}(f(a, a, c)+f(b, b, c)) \leq f(a, b, c) .
\end{aligned}
$$

In (2.7) the first two inequality signs are due to Jensen's inequality and the last one arises by using property (2.6). We conclude that $f$ has the property of minimum isosceles extrema.

Remark As we shall show below both the above sufficient conditions, namely (1.2) and that from Proposition 2, are not as good as our main result Theorem 1. There are functions depending on the triangle's elements that satisfy neither (1.2) nor Proposition 2 but they do obey the PIT.

\section{Illustrations}

\subsection{The fundamental inequality of a triangle}

Here we want to illustrate the importance of our result. Our main aim is to establish that the vast majority of the theory of geometric triangle inequalities can be obtained from our main result Theorem 1 . We do this by establishing first the fundamental inequality of a triangle $[4$, p. 2]. Thereafter, this will imply that the method will work for any equivalent inequality formulated with three independent elements of a triangle. Furthermore, we show that the principle of the isosceles triangle also holds for geometric inequalities that involve possibly some elements outside those given from the triangle elements themselves. This will be illustrated by the second application through establishing one well-known result of Paul Erdös. Finally, as a direct illustration of Corollary 3, we give a somewhat unexpected application of the main result, illustrating a direct connection between the class of general triangles with that of the isosceles ones. All these applications are pointing towards the universality of the principle of the isosceles triangle as being the fundamental result in triangle geometry.

We start by showing how one can use our result to establish the fundamental inequality for a triangle. In [4, p. 2] the following result of E. Rouché (1851) is stated:

Theorem 2 (The fundamental inequality for a triangle). A necessary and sufficient condition for the existence of a triangle with elements $r, R$ and $p$ is

$$
2 R^{2}+10 R r-r^{2}-2(R-2 r) \sqrt{R^{2}-2 R r} \leq p^{2}
$$

and

$$
2 R^{2}+10 R r-r^{2}+2(R-2 r) \sqrt{R^{2}-2 R r} \geq p^{2}
$$

where $r, R$ are the radii of the inscribed and circumscribed circles, respectively, and $p$ is the semiperimeter. Furthermore, if one of the signs in (3.1) or (3.2) is equality then the triangle is isosceles and vice-versa. 
Proof. We start with the necessary condition. We have to show that (3.1) and (3.2) hold in every triangle. To do this, we apply the lemma and Theorem 1. Consider the following functions:

$$
\begin{aligned}
& h_{1}(a, b, c)=p^{2}-2 R^{2}-10 R r+r^{2}+2(R-2 r) \sqrt{R^{2}-2 R r}, \\
& h_{2}(a, b, c)=-p^{2}+2 R^{2}+10 R r-r^{2}-2(R-2 r) \sqrt{R^{2}-2 R r} .
\end{aligned}
$$

By using standard trigonometric identities, one can check that $h_{1}(\pi-2 t, t, t)=0$ for all $0 \leq t \leq \frac{\pi}{3}$ and is strictly positive for $\frac{\pi}{3}<t \leq \pi$. Similarly, we have that $h_{2}(\pi-2 t, t, t)>$ 0 for all $0 \leq t \leq \frac{\pi}{3}$ and $=0$ for $\frac{\pi}{3}<t \leq \pi$. This establishes that the assumption (2.1) of the lemma is satisfied for $h_{1}$ and $h_{2}$. We now want to check the property of global isosceles extrema for $h_{1,2}$. We do this only for $h_{1}$ here. For $h_{2}$ it can be done in a similar way.

First, on using standard results from triangle geometry $(r=4 R \sin (a / 2) \sin (b / 2) \sin (c / 2)$ and $p=R(\sin (a)+\sin (b)+\sin (c)))$ we can reduce the problem to show that

$$
\begin{aligned}
& h_{1}(a, b, c)=(\sin (a)+\sin (b)+\sin (c))^{2}-2-40 \sin (a / 2) \sin (b / 2) \sin (c / 2) \\
& +16 \sin ^{2}(a / 2) \sin ^{2}(b / 2) \sin ^{2}(c / 2)+2(1-8 \sin (a / 2) \sin (b / 2) \sin (c / 2))^{3 / 2} \geq 0
\end{aligned}
$$

for all $a, b, c \geq 0, a+b+c=\pi$. In order to apply Theorem 1, we take $c>0$ (the case $c=0$ is trivial) and introduce the function $h_{1}^{+}(x)=h_{1}(a+x, b-x, c)$ for $0 \leq x \leq$ $\min \{\pi-a,|b-c|\}$. It is straightforward to check that

$$
\begin{aligned}
& \left.\frac{\mathrm{d} h_{1}^{+}}{\mathrm{d} x}\right|_{x=0}=4 \sin \left(\frac{a-b}{2}\right)(5 \sin (c / 2)-\cos (c / 2)(\sin (a)+\sin (b)+\sin (c))) \\
& \quad-4 \sin (a / 2) \sin (b / 2) \sin ^{2}(c / 2)+3 \sin (c / 2) \sqrt{1-8 \sin (a / 2) \sin (b / 2) \sin (c / 2)} .
\end{aligned}
$$

The last expression is zero only if either one of the two factors in (3.6) is zero. This gives that either $a=b$ (from $\sin ((a-b) / 2)=0$ ) or that the second factor is zero. Denote by $h h_{1}(a, b, c)$ the factor multiplying $4 \sin \left(\frac{a-b}{2}\right)$ in (3.6). We will show that $h h_{1}$ is positive in the set of all triangles and is zero only when the triangle is isosceles. Indeed, for $h h_{1}$ it is again straightforward to check that the hypotheses (2.1) in the lemma are satisfied. For the second hypothesis (i.e. the minimum isosceles extremum property) we are using Theorem 1. Therefore, let us compute

$$
\left.\frac{\mathrm{d} h h_{1}}{\mathrm{~d} x}\right|_{x=0}=2 \sin \left(\frac{a-b}{2}\right)\left(\sin ^{2}(c / 2)+\cos ^{2}(c / 2)+\sin ^{2}(c / 2)(1+3 / P)\right)
$$

where $P=\sqrt{1-8 \sin (a / 2) \sin (b / 2) \sin (c / 2)} \geq 0$ (see [4]). It follows that the expression in (3.7) is zero only when $a=b$ (the parenthesis on the right-hand side member of (3.7) is positive). This shows that Theorem 1 applies and we deduce that $h h_{1}$ is zero only when $a=b$. A further application of Theorem 1 for $h_{1}^{+}$via (3.6) then establishes the result that $h_{1}^{+}$has the property of global isosceles extrema. These considerations then establish inequality (3.1). Similarly, one can show that (3.2) is valid.

For the sufficiency part one needs only note that relations (3.1) and (3.2) guarantee that the cubic in $z, h(z)=z^{3}-2 p z^{2}+\left(p^{2}+r^{2}+4 R r\right) z-4 p R r=0$ has three positive 
roots which satisfy the conditions for being the sides of a triangle ([4, pp. 6-7]). This establishes the theorem. We note that the proof in [4] does not contain reference to why there is equality in (3.1) and (3.2) when the triangle is isosceles. Based on Theorem 1 this is now obvious.

Remark In [4, pp. 7-18] there is a long list of equivalent formulations of the fundamental triangle inequality in terms of other elements of the triangle. Based on our main result Theorem 1 it is straightforward to establish that each of them holds in the same way as above.

Application 1. By applying the same method as above the lemma and Theorem 1 immediately give that in every triangle we have the following inequalities:

$$
\begin{aligned}
3 \sqrt{3} r \leq p & \leq 2 R+(3 \sqrt{3}-4) r, \\
16 R r-5 r^{2} & \leq p^{2} \leq 4 R^{2}+4 R r+3 r^{2} .
\end{aligned}
$$

W.J. Blundon was the first to establish that (3.8) is the best inequality linear in $r$ and $R$ [1]. The situation for the quadratic case is more subtle, and, as discussed in [4], it turns out that (3.9) is only one representative of a family of such possible "best inequalities". Very recently we have extended Blundon's optimality results for the linear and quadratic case, to a general theorem for all degrees, which generalises many of the previous cases known in the literature [9].

Theorem 3. In every triangle $A B C$ we have the inequality

$$
\alpha^{n}+\beta^{n}+\gamma^{n} \leq 2^{1+n} R^{n}+2^{n}\left(3^{1+n / 2}-2^{1+n}\right) r^{n}
$$

for all $n \geq 0$ where $\alpha, \beta, \gamma$ represent the lengths of the sides of the triangle $A B C$. The constants on the right-hand side are the best possible for each $n \geq 0$.

We now compare the PIT method with the two sufficient conditions given at the end of Section 2. For example, let us analyse the Application 2.3 from [8].

Application 2. Given an acute triangle, let $h_{a}, h_{b}, h_{c}$ denote, respectively, its altitudes, and let $p$ denote its semiperimeter. Show that

$$
\sqrt{3} \max \left\{h_{a}, h_{b}, h_{c}\right\} \geq p .
$$

This is problem 10418 [6, p. 1013] proposed by the author. As in [8], (3.11) can be transformed to the equivalent form

$$
f(a, b, c)=2 \sqrt{3} \sin \left(\frac{a}{2}\right) \sin \left(\frac{b}{2}\right)-\cos \left(\frac{c}{2}\right) \geq 0 \text { for } 0 \leq c \leq b \leq a \leq \frac{\pi}{2} .
$$

We shall verify only the condition for minimum isosceles extrema given by Theorem 1. Consider $f_{-}(x)=f(a-x, b+x, c)$. Then it is easy to check that $\left.f_{-}^{\prime}(x)\right|_{x=0}=$ $\sqrt{3} \sin \left(\frac{a-b}{2}\right)$. Therefore, $f_{-}^{\prime}(0)=0 \Leftrightarrow a=b$ and the property is established. In [8] (3.11) was established by checking that condition (1.2) was satisfied by $f$ in (3.12), which is a 
considerable more difficult task. Also Proposition 1 does not work in this case. Indeed, a simple calculation gives that

$$
2 f(a, b, c)-f(a, a, c)-f(b, b, c)=-2 \sqrt{3}(\sin (a / 2)-\sin (b / 2))^{2}<0 .
$$

The following example shows that PIT holds even if (1.2) is violated. For example, consider the function

$$
f(a, b, c)=\frac{3}{2}(\cos (b)+\cos (c))-\cos \left(\frac{b-c}{2}\right)-\sin \left(\frac{a}{2}\right)
$$

for $0 \leq c \leq b \leq a \leq \pi, a \geq \pi / 2$, and $a+b+c=\pi$. It can be easily checked that $f(a, b, c)-f\left(a, \frac{b+c}{2}, \frac{b+c}{2}\right)=\left(3 \sin \left(\frac{a}{2}\right)-1\right)\left(\cos \left(\frac{b-c}{2}\right)-1\right)<0$. Therefore, (1.2) is not satisfied. However, a simple calculation shows that for $f_{-}(a, b,-x, c+x)$ we have $f_{-}^{\prime}(0)=\sin \left(\frac{b-c}{2}\right)\left(3 \sin \left(\frac{a}{2}\right)-1\right)$, which is zero only for $b=c$. Furthermore, $f$ is positive when restricted to only the class of isosceles triangles such that $b \leq \pi / 3$.

We note that the vast majority of the geometric inequalities involving just some or all the important elements of a triangle can be deduced, verified and indeed established as a straightforward application of the lemma and Theorem 1. In this respect, the principle of the isosceles triangle plays a universal character.

\subsection{The Erdös-Mordell-Barrow inequality}

We now show how one can extend the principle of the isosceles triangle so as to deal with inequalities, which may involve explicitly external elements to the triangle itself. For example, one may want to establish bounds between the distances from an arbitrary point in the triangle to its vertices or its sides and possibly compare these to other known elements of the triangle (sides, altitudes, etc.). To illustrate the ideas, we shall present as a typical application the following well-known result due to Erdös [2] and later proved by Mordell and Barrow [5], see also [3] for a recent proof.

Theorem 4 (Erdös-Mordell-Barrow). Let $A B C$ be a triangle and $P$ a point inside it. Denote by $d_{a}, d_{b}, d_{c}$ the distances from $P$ to the triangle's sides. Then

$$
P A+P B+P C \geq 2\left(d_{a}+d_{b}+d_{c}\right) .
$$

Proof. We start by following the standard method and note that the following inequality holds true $A B \cdot P A \geq C A \cdot d_{b}+B A \cdot d_{c}$ (and the other two obtained by circular symmetry) for any point $P$ inside $A B C$ [4]. By taking the symmetrical point $P^{\prime}$ to $P$ with respect to the bisector of the angle $B A C$ one also has $A B \cdot P^{\prime} A \geq C A \cdot d_{c}+B A \cdot d_{b}$ valid [3]. Now, we depart from the proof in [3] and simply apply the crucial property related to the principle of the isosceles triangle. It is easy to observe that the "best" inequalities as above will be obtained when $A P=A P^{\prime}$ but this will correspond to the triangle $A B C$ being isosceles. In other words, the function that maps a point $P$ to the difference $A B \cdot P A-\left(C A \cdot d_{c}+B A \cdot d_{b}\right)$ has the minimum isosceles property (here due to symmetry considerations with respect to the angle bisector). Similar reasoning related to the other sides shows that the best (i.e. closest) inequality will be obtained for the case when the triangle will be isosceles two by 
two sides thus being equilateral. However, it is straightforward to see that (3.14) holds true (and in fact it is an equality) in such a case, which ends the proof of the result.

Remarks Although the above proof is not simpler than the standard proof by Barrow it does underscore the essential property of the Erdös-Mordell inequality that its strongest case is given by the case of an isosceles triangle. The above ideas can be easily applied to establish similar results, for example, to obtain the various generalisations and extensions of the Erdös-Mordell theorem. Many examples and extensive discussions are given in [4, Chap XI, pp. 313-319]. In fact, we published recently the following new result related to the Erdös-Mordell theorem [7].

Application. With the above notations the following inequalities hold in every triangle

$$
\begin{gathered}
k^{P A}+k^{P B}+k^{P C}+3 \geq 2\left(k^{d_{a}}+k^{d_{b}}+k^{d_{c}}\right), \\
k^{P A+P B}+k^{P B+P C}+k^{P C+P A}+6 \geq k^{2 d_{a}}+k^{2 d_{b}}+k^{2 d_{c}}+2\left(k^{d_{a}}+k^{d_{b}}+k^{d_{c}}\right)
\end{gathered}
$$

for any $k \geq 1$. See [7] for details.

\subsection{A connection between non-isosceles and isosceles triangles}

We end this section by giving a rather unusual and a priori unexpected application of the principle of the isosceles triangle. This application is an illustration of Corollary 3 above.

Proposition 2. For any non-isosceles triangle there is at least one isosceles triangle having the same perimeter and area as well as equal inscribed and circumscribed radii.

Proof. Let $\Delta=X Y Z$ (with $X \geq Y \geq Z$ ) be a non-isosceles triangle of semiperimeter $p$, area $S$ and having the radius of the circumscribed circle equal to $R$. Let also $r$ be the radius of its inscribed circle. It is straightforward to verify that the function $g: \Delta \rightarrow \mathbb{R}^{3}$, $g(a, b, c)=(p, S, R)$ satisfies the requirements in Corollary 3. Define by $\Delta(t)$ the set of all isosceles triangles with the angles of the form $X(t)=Y(t)=t, Z(t)=\pi-2 t$, $\frac{\pi}{2} \geq t \geq \frac{\pi}{3}$. Now, consider the function

$$
h: \Delta(t) \rightarrow \mathbb{R}^{3}, \quad h(t)=(p-p(t), S-S(t), R-R(t)),
$$

where $p(t), S(t)$ and $R(t)$ are the perimeter, area and the circumscribed radius of $\Delta(t)$, respectively. Again, it is straightforward to check that $h$ satisfies the hypotheses of Corollary 3 . Therefore, we deduce that there is some $t_{0}$ with $\frac{\pi}{2} \geq t_{0} \geq \frac{\pi}{3}$ such that $h\left(t_{0}\right)=0$. If we note that for every triangle one has $S=p r$ this ends the proof.

\section{Conclusions}

There are many methods of proving geometry by calculus and in some cases they do not always give a real insight into the geometrical aspects of the result. However, although it is not a statement of a theorem, we argue that the principle of the isosceles triangle (PIT) is a fundamental result on which an extensive part in the field of geometric triangle inequalities can be understood and learned. We hope that we have made this sufficiently clear through 
the presentation of the various applications (and their many corollaries and extensions) in the previous section. For example, most of the material in the book [4], which is the standard reference in the field, can be easily established in this way.

In an abstract way, the essence of our main result can be summarised as follows. For a given function $g$ on $T$ (as in the PIT lemma) the solutions of the equation $g=0$ in $T$ giving the global minimum under a certain group action or set of symmetries (the set of the isosceles triangles, for example) remain invariant when the action is extended to a bigger class (here the set of all triangles).

We hope that the principle of the isosceles triangle presented here will help enrich the field of geometric inequalities by opening up a systematic method for establishing old and new inequalities.

\section{Acknowledgements}

The author is grateful to M. Lackenby (Oxford) for suggesting example (2.2), C.P. Niculescu (Craiova) and to the referee for useful comments.

\section{References}

[1] Blundon, W.J.: Inequalities associated with the triangle. Canad. Math. Bull. 8 (1965), 615-626.

[2] Erdös, P.: Proposed problem 3740. Amer. Math. Monthly 42 (1935), 396.

[3] Komornik, V.: A short proof of the Erdös-Mordell Theorem. Amer. Math. Monthly 104 (1997), 57-60.

[4] Mitrinovic, D.S.; Pecaric, J.E.; Volenec, V.: Recent Advances in Geometric Inequalities. Kluwer Academic, Dordrecht-Boston-London 1989.

[5] Mordell, L.J.; Barrow, D.F.: Solution of Problem 3740. Amer. Math. Monthly 44 (1937), 252-254.

[6] Satnoianu, R.A.: Proposed problem 10418. Amer. Math. Monthly 101 (1994), 1013.

[7] Satnoianu, R.A.: Proposed problem 10814. Amer. Math. Monthly 107 (2000), 567.

[8] Satnoianu, R.A.: A universal method for establishing geometric inequalities in a triangle. Amer. Math. Monthly 108 (2001), 360-364.

[9] Satnoianu, R.A.: General power inequalities between the sides and the circumscribed and inscribed radii related to the fundamental triangle inequality. Math. Inequal. Appl. 5 (2002), 745-751.

Razvan Alin Satnoianu

Department of Mathematics

City University London

London EC1V 0HB, UK

e-mail: r.a.satnoianu@city.ac.uk 